\title{
Periglaziäre, polygonal-verzweigte rinnenförmige Bildungen und glazitektonische Strukturen in Saale-Till am Elbe- Urstomtalrand bei Wedel [Schleswig-Holstein]
}

\author{
Alf Grube
}

How to cite:

Grube, A. (2015): Periglaziäre, polygonal-verzweigte rinnenförmige Bildungen und glazitektonische Strukturen in Saale-Till am Elbe-Urstomtalrand bei Wedel (Schleswig-Holstein). - E\&G Quaternary Science Journal, 64 (1): 3-13. DOI: 10.3285/eg.64.1.01

Kurzfassung:

\begin{abstract}
Während der Sanierung eines Raffineriegeländes in Wedel / Holstein wurden im Jahre 2008 auffällige Strukturen beobachtet. Die in Aufsicht polygonal verzweigten, rinnenartigen Strukturen sind in einen bindigen und kalkreichen, saalezeitlichen Till der Niendorf-Formation eingeschnitten und im Querschnitt symmetrisch halbkreisförmig ausgebildet. Die polygenetisch entstandenen Strukturen sind mit kalkhaltigen, schluffigen Sanden gefüllt, die als umgelagerter Till mit aus den hangenden Sanden vermischtem Material bzw. äolischen Komponenten interpretiert werden können. Die Breite der Strukturen beträgt ca. 0,3 bis $1,5 \mathrm{~m}$, die Tiefe bis zu ca. 0,8 m. Bis zu mehr als $10 \mathrm{~m}$ lange Risse, als schmale Bänder rötlich gefärbter Sande ausgebildet, sind oft zentral in den o. g. rinnenartigen Strukturen positioniert. Die rötliche Färbung ist auf anthropogene Verunreinigung zurückzuführen. Diese Bänder reichen bis auf die Sohle der rinnenartigen Formen. An der Unterseite der Rinnenstrukturen setzen häufig glazitektonisch angelegte Klüfte an, die bis zu wenige Zentimeter breit sind und über mehrere Meter Tiefe bis an die Sohle der Auskofferung zu verfolgen waren. Die Genese der Strukturen, die einen möglichen Zusammenhang der rinnenartigen Strukturen mit dem glazitektonischen Kluftnetz bzw. den vermuteten paläohydrogeologischen Verhältnissen einschließt, wird diskutiert.
\end{abstract}

Periglacial polygonal-dendritic channelized forms and glacitectonic structures in Saalian till at the rim of the Elbe spillway in Wedel [Schleswig-Holstein]

\begin{abstract}
Remarkable polygenetical structures were observed at a refinery rehabilitation site in Wedel/Holstein. The polygonal shaped, channel-like structures are incised in mid-Saalian clayey, chalk-rich till. They are symmetrically semicircular shaped and filled with calcareous, silty sands that can be interpreted as sandy reworked till with aeolian components. The width reaches from approx. 0.3 to 1.5 metres, the depth up to approx. 0.8 metres. Horizontal cracks up to more than 10 meters in lengths, occurring as narrow belts of sand with reddish colour, are often centrally aligned in the channel-like structures. The present-day red colour is not natural but related to pollution. These belts reach down to the bottom of the channel like structures. At the lower site of the channel-like structures glacitectonic fissures with a width up to a few centimetres were to be traced into a depth of several metres to the basis of the excavat ion. The genesis of the channel-like structures is discussed. e. g. a possible relation with the pre-existing glacitectonic joints resp. the expected periglacial paleohydrogeological setting.
\end{abstract}

Keywords: periglacial channels, frost wedges, glacitectonism, paleohydrogeology, talik, Weichselian periglacial, Elbe spillway

Address of author: $\quad$ A. Grube, Geologisches Landesamt, Behörde für Umwelt und Energie, Neuenfelder Straße 19, 21109 Hamburg, E-Mail: alf.grube@bsu.hamburg.de

\section{Einleitung}

Im Frühjahr 2008 wurden bei der Sanierung eines Raffineriestandortes in Wedel (Holstein) auffällige Sedimentstrukturen beobachtet. Diese waren in einem Aufschluss auf dem Gelände der ehemaligen Raffinerie (ExxonMobil; Abb. 1) sichtbar. Der Geologische Dienst Schleswig-Holstein wurde um eine Expertise gebeten. Da ein tiefer liegender Grundwasserleiter mit Schadstoffen (MKW, PAK, CKW / LHKW, BTEX, teilweise in Phase; vgl. BIG 2009) belastet ist, sollte geklärt werden, ob die unten beschriebenen
Strukturen, neben der nicht ganz auszuschließenden Möglichkeit unfachmännisch ausgebauter GW-Messstellen, für eine hydraulische Verbindung zwischen den durch einen mächtigen Grundwassergeringleiter getrennten Grundwasserleitern, bzw. eine entsprechende Verunreinigung verantwortlich sein könnten. Die geologischen Aufnahmen erfolgten im Beisein von Herrn Seifert (ARCADIS) in einem kontaminierten Bereich („Hotspot 1“), unter Einhaltung spezieller Arbeitsschutzmaßnahmen (Schutzanzug, eng begrenzte Aufenthaltszeit, usw.). Neben der Freilegung von Strukturen an der Sohle und an den bis zu 4 m hohen 


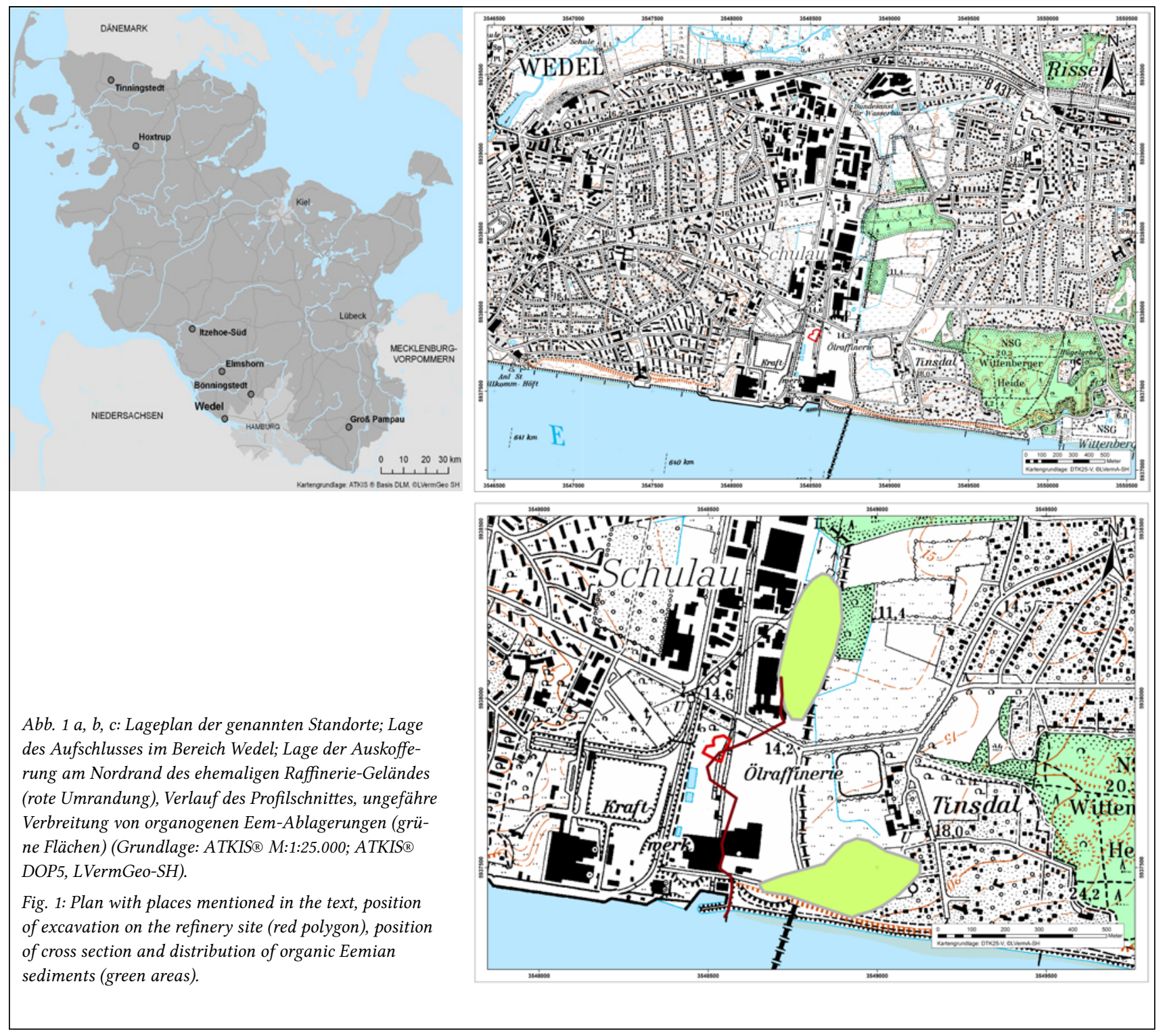

Wänden der Auskofferung, wurden aus den kontaminierten Bereichen Proben für Korngrößenanalysen und Kalkgehaltsbestimmungen entnommen und diese durch die Fa. ARCADIS zur Untersuchung gegeben. Umfangreichere Geländeuntersuchungen waren aufgrund der erheblichen Kontamination mit Schadstoffen und der nur kurzfristig bestehenden Aufschlüsse nicht möglich. Eine genaue Aufnahme der Strukturen hinsichtlich ihrer Form und flächenhaften Ausdehnung war aufgrund der geringen Ausdehnung der Schurf-Fläche nicht durchführbar. Trotz dieser Einschränkungen erschien es dem Autor sinnvoll, diese bisher in der Literatur nicht beschriebenen Strukturen mitzuteilen. Im Folgenden wird aus praktischen Gründen für den eigentlich korrekterweise zu verwendenden Ausdruck „rinnenartige, mit bindigem Material verfüllte, polygonal verzweigte Strukturen“ der Begriff „Rinnen-Polygone“ verwendet.

\section{Geologie und Geomorphologie des Untersuchungs- gebietes}

Das Untersuchungsobjekt liegt im Geest-Bereich, unmittelbar westlich der Stadtgrenze zu Hamburg (Abb. 1). Es liegt randlich zum Steilufer des Elbe-Urstromtales, welches hier eine Höhe von 10-12 m erreicht. Als älteste Schichtglieder sind ein vermutlich während der Elster-Kaltzeit gebildeter Till sowie hangender Lauenburger Ton in Bohrungen aufgeschlossen (Abb. 2). Die hangende Schichtenfolge ist geprägt durch einen Unteren und einen Oberen Till, dessen Zwischenmittel ein bis zu $2 \mathrm{~m}$ mächtiger, glazifluviatiler Sand bildet. Der Untere Till dürfte nach der ortsüblichen Einordnung (GRUBE 1981; STEPHAN 1995) aufgrund der im Feld erkennbaren petrographischen Merkmale der Drenthe-Formation angehören (Ältere Saale). Dieser setzt sich in südlicher Richtung unter der Elbe fort. Der obere Till ist im Bereich des Elbe-Urstromtales vollständig ausgeräumt worden. Er wird hier der Mittleren Saale zugeordnet (Niendorf-Formation im Hamburger Raum, Hennstedt-Vorstoß in Schleswig-Holstein; siehe auch nächster Abschnitt). Die Schichtenfolge wurde durch glazitektonische Vorgänge erheblich beansprucht. Die Bereiche Wedel und östlich der Landesgrenze Schleswig-Holstein/Hamburg sind für ihre teilweise intensive glazitektonische Beeinflussung seit langer Zeit bekannt (WolfF 1913; Wilke 1984; Wilke \& EHLERS 1984; NitSCHE 2012). Wilke (1984) konnte benachbart eine Verstellung bis in eine Teufe von mehr als $100 \mathrm{~m}$ nach- 
weisen. Für diesen Bereich ist eine intensive Verschuppung nachgewiesen. Nachweise von intensiver Glazitektonik wurden auch durch aktuelle Bohrungen im Bereich des Industriebetriebes Möller geführt. In den letzten Jahren ausgehobene größere Baugruben im zentralen Wedel zeigten vorwiegend eine intensive glazitektonische Überprägung in Form von komplizierten Störungs-Sytemen in den ansonsten verhältnismäßig wenig gestörten Ablagerungen.

Im Südostbereich der ehemaligen Raffinerie-Fläche finden sich im Liegenden rolliger Ablagerungen bis $\mathrm{zu}$ ca. 2 Meter mächtige, organogene Eem-Sedimente. Diese füllen die östlich gelegenen, ehemaligen Talformen aus. Das Schulauer Ufer wurde bereits von Charles Lyell untersucht (vgl. F. GRUBE 1962). Die Eem-Ablagerungen liegen dabei meist direkt dem Niendorf-Till auf. In tieferen Senken treten zwischengeschaltet auch geringmächtige Sande auf. Eine pollenanalytische Datierung der Eem-Ablagerungen wurde von Hallik (1957, vgl. Lit. bei GrubE $1962 \mathrm{zu}$ weiteren Bearbeitern) veröffentlicht. Auch nördlich des Raffinerie-Geländes wurden unmittelbar oberhalb des Tills (Toteissenken?) humose Sedimente angetroffen, die vermutlich ebenfalls in die Eem-Warmzeit einzustufen sind (Abb. 1; Abb. 2). Im Hangenden des Oberen Tills, bzw. der Eem-Sedimente folgen mehrere m mächtige Sande mit fluviatiler und äolischer Herkunft. Die Sande im Hangenden des Niendorf-Tills wurden während der mittleren Saale (Nachschüttsande), der jüngeren Saale und / oder der Weichsel-Kaltzeit gebildet (Tab. 1). Eine genaue Zuordnung ist nicht möglich. Neuere flächenhafte Bearbeitungen der Schichtenfolge (NITSCHE 2012) lassen es als wahrscheinlich gelten, dass der Großteil der Weichsel-Kaltzeit zuzuordnen ist. Als oberste Einheit in der ehemals unbeeinflussten Sedimentabfolge finden sich Flug- und Dünensande. Benachbart sind entsprechende ausgedehnte äolische Flächen im Dünengebiet Wittenbergen vorhanden.

Der Schadstoff-Hotspot, in dem die hier diskutierten Strukturen abschnittsweise freigelegt wurden, hatte eine flächenhafte Ausdehnung von ca. 50 x 70 m. Der Aufschluss zeigte eine flächige Verbreitung eines homogenen, bindigen, kreide- und flintreichen, grauen Tills, welcher der Mittleren Saale zugeordnet werden kann. Es handelt sich um einen schluffigen Sand mit einem Kalkgehalt von 10-15 \%. Die Oberfläche dieses Tills ist unregelmäßig. Das Polygonnetz (Abb. 4, 5) ist jedoch nicht in Streifenform verzerrt, sodass eine stärkere Hangneigung keinen Einfluss gehabt haben kann. Die Till-Oberfläche zeigt nach Informationen der Fa. ARCADIS meist Werte zwischen $+5,5$ und $+6,5 \mathrm{~m} \mathrm{NN}$, im östlichen Bereich des Hotspots fallen diese insgesamt ab. Es zeigen sich keine deutlichen Anzeichen für Eis-Segregation in den oberen Abschnitten des Tills. Die auf den Till folgenden hangenden Sande waren im ausgekofferten Bereich zum Zeitpunkt der Begehungen nicht aufgeschlossen (Spundwand). Im Liegenden des Tills folgt ein geringmächtiger Grundwasserleiter, unterhalb erneut Till (Abb. 2).

\section{Feldbeobachtungen}

Die auffälligste Erscheinung im Aufschluss waren zunächst teilweise verzweigt angeordnete Rinnen (Abb. 3, 4). Aufgrund der nur kleinräumigen Auskofferung waren diese auf wenigen hundert Quadratmetern aufgeschlossen. Bei den Strukturen handelt es sich um rinnenartige Vertiefungen, die mit kalkhaltigen, sandigen Schluffen gefüllt (Korngrößenanalyse Fa. ARCADIS) und in den kreidereichen, bindigen Till eingeschnitten sind. Der umgebende Till ist, bis auf dünne Klüfte (siehe unten), homogen ausgebildet und ungestört. Die Rinnen-Polygone sind unregelmäßig bezüglich ihrer Form und Breite. Die Breite beträgt ca. 0,3 bis $1,5 \mathrm{~m}$, die Tiefe bis zu ca. 0,6 m (Abb. 3-5). Sie bilden ein polygonartiges Netz, die Weite der Polygone liegt bei max. wenigen Metern. Die Kontinuität des Verlaufs der Rinnen-Polygone, bzw. des Polygonnetzes in die Fläche hinein, konnte aufgrund der Aufschlussverhältnisse nur lokal erfasst werden. Die Form der Oberfläche, bzw. der Kontaktbereich zu den hangenden Sanden war aufgrund der Auskofferung nicht zu erfassen.

Die Füllung der Rinnen-Polygone ist verhältnismäßig homogen zusammengesetzt. Meist ist das Material sandigschluffig, die Hauptbodenart wird aus Fein- bis Mittelsand gebildet. Es ist keine Schichtung oder anderweitige deutliche interne Gliederung zu erkennen. Es sind jedoch kleine

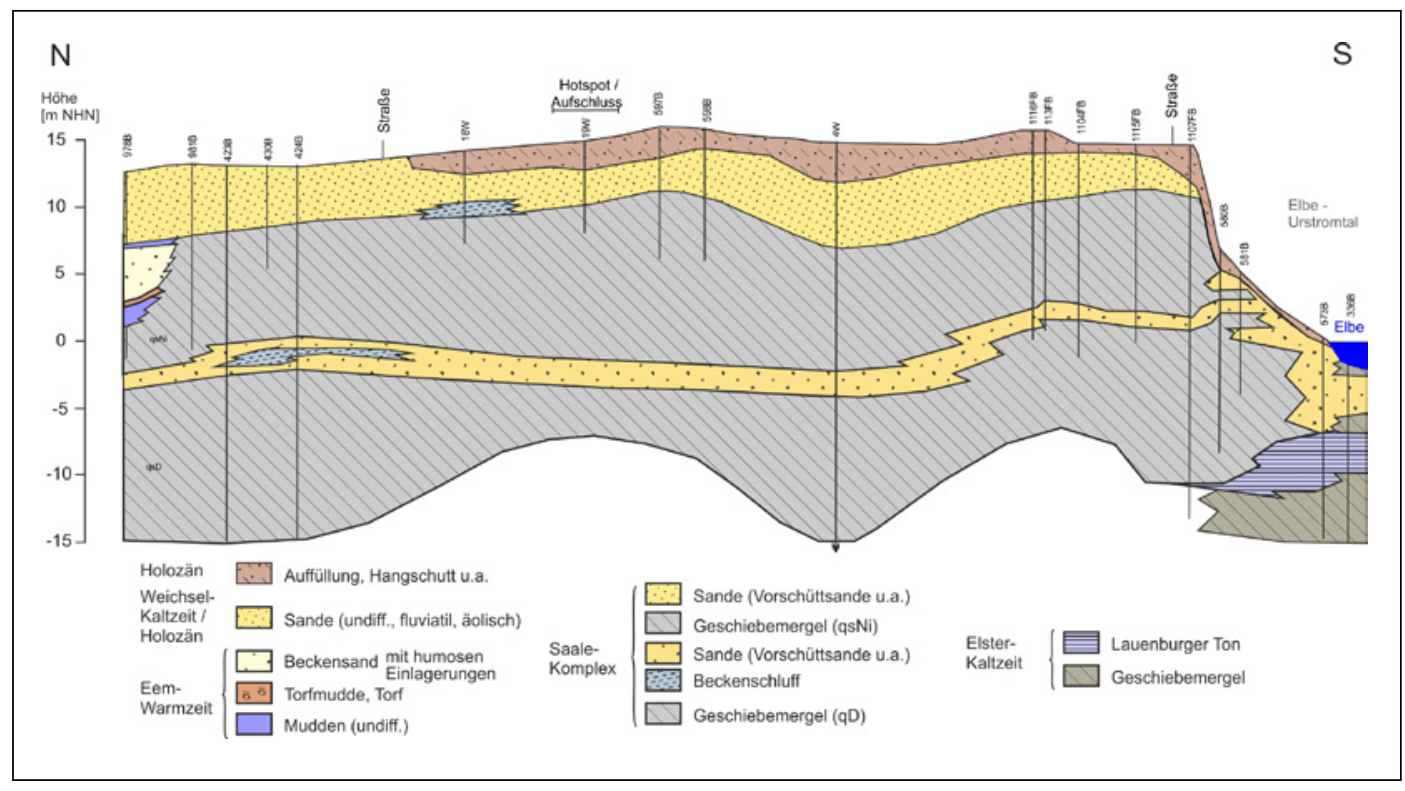

Abb. 2: Profilschnitt durch den Untersuchungsraum, 10-fache Überhöhung. (Daten: Geologisches Landesarchiv LLUR; W. BOCK; K.-H. NACHTIGALL; 7 . NIEDERMAYER; EXXONMobil / ARCADIS).

Fig.2: Cross section through investigation area, exaggeration $10 x$ (data: Geological Archive LLUR; W. BOCK; K.-H. NACHTIGALL; f. NIEDERMAYER; ExxonMobil / ARCADIS). 


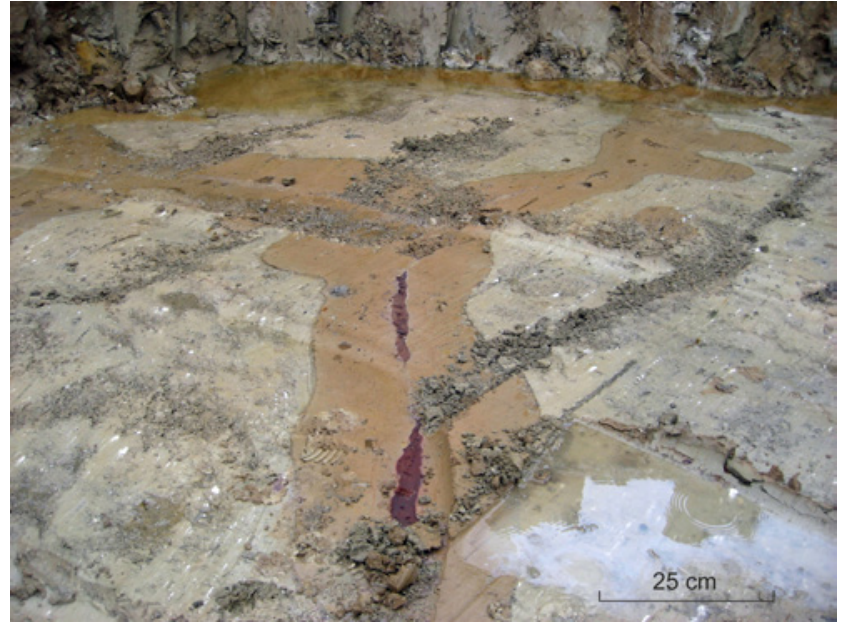

Abb. 3: Polygonal verzweigte, rinnenartige Strukturen, die in den zentralen Bereichen rot gefärbte Sande enthalten (Photo: S. Seifert, Fa. ARCADIS). Breite der Rinnen beträgt ca. 0,6 m.

Fig. 3: Periglacial polygonal-dendritic channelized structures, with central red coloured sands (Photo: S. Seifert, Fa. ARCADIS). Widths of structures ca. 0,6 m.

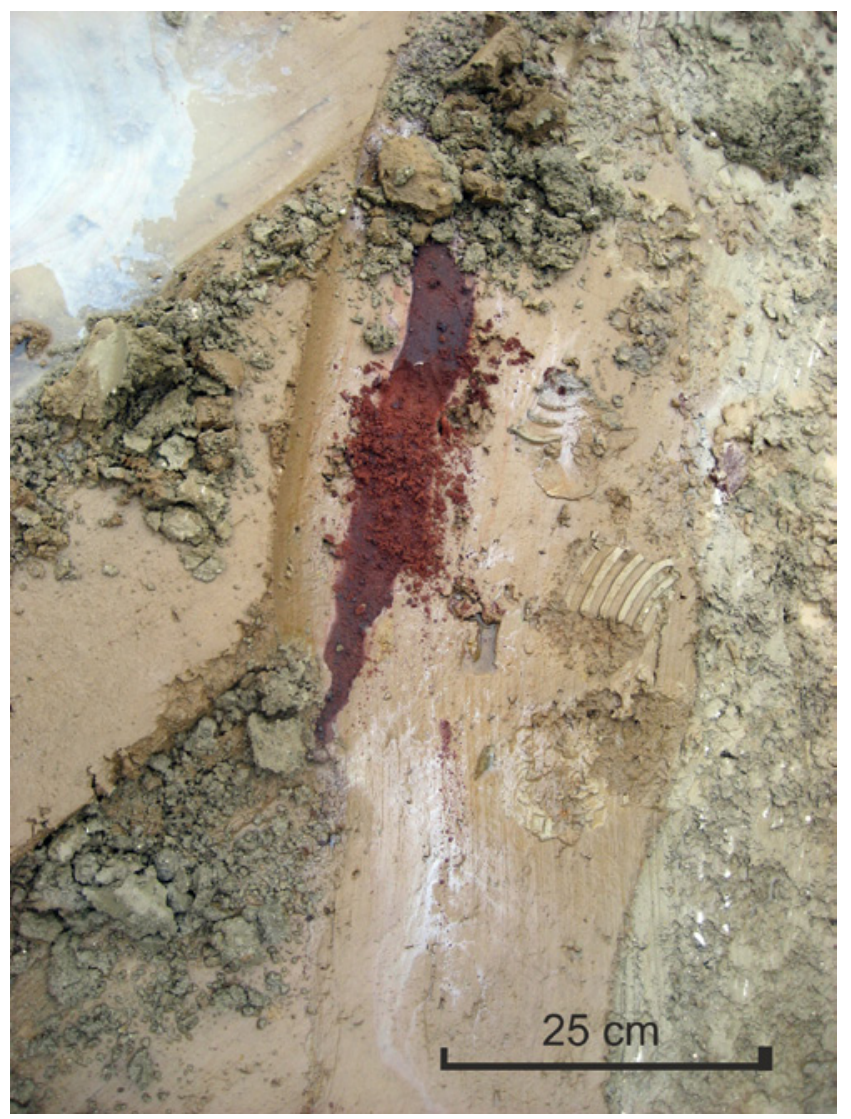

Abb. 4: Rinnenartige Struktur mit breitem, unregelmäßigem Streifen rot gefärbter Sande (Photo: S. Seifert, Fa. ARCADIS). Länge des rot gefärbten Streifens ca. 0,4 $\mathrm{m}$.

Fig. 4: Detail photo of polygonal channelized structures with central red coloured sands (Photo: S. Seifert, Fa. ARCADIS). Lengths of red coloured band ca. $0,4 \mathrm{~m}$.

Sandeinschaltungen (Gerölle?) sowie lokale, geringmächtige Sandeinschaltungen an der Basis erkennbar (Abb. 5). Die Korngrößenunterschiede zwischen dem umgebenden Till und der Füllung der Rinnen-Polygone sind gering. Der

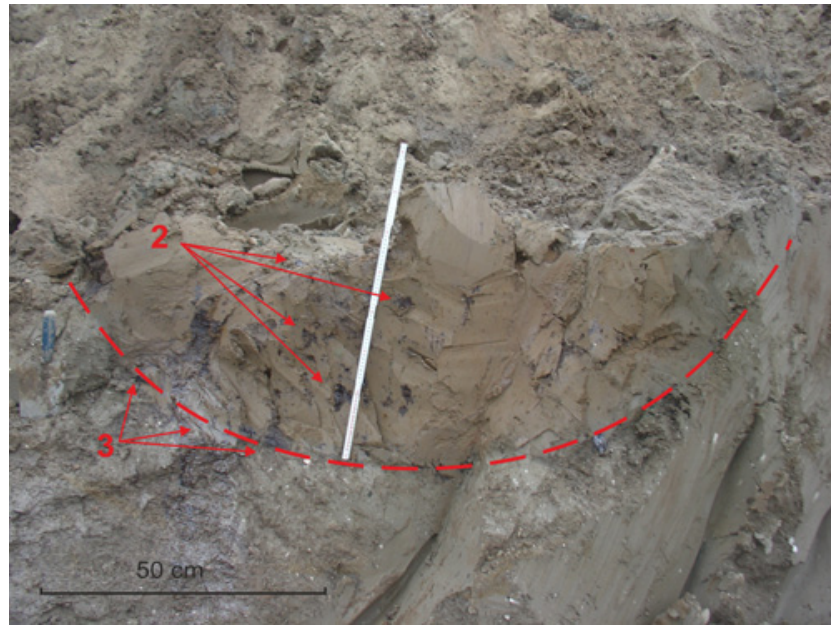

Abb. 5: Rinnen aus Abb. 3 und 4 im Querschnitt (Zollstock ca. 0,8 $\mathrm{m}$ lang). Erkennbar sind die gegenüber dem umgebenden Till abweichende Korngrößenverteilung, die Inhomogenitäten innerhalb der Rinnenfüllung (die zu erhöhter hydraulischer Durchlässigkeit führen) mit Austritt von Teerölen (2) sowie die verstärkte Schadstoffführung an der Unterkante der Struktur (3). Tiefe der Rinne ca. 0,7 m.

Fig. 5: Channels from fig. 3 and 4 in cross section (folding rule ca. 0,8 m long). Visible is the deviation of grain size compared to surrounding till, the inhomogeneity inside channel filling (leads to accelerated hydraulic permeability) with discharge of creosote (2) and increased occurrence of pollutants at the base of structure (3). Depths of channel ca. 0,7 m.

Kalkgehalt liegt bei ca. 5,3\% und ist damit deutlich niedriger als im umgebenden Till. Örtlich sind Inhomogenitäten aus gröberen Einschaltungen vorhanden.

Es treten Brodelboden-artige Strukturen im ursprünglichen Till, d.h. außerhalb der Rinnen-Polygone, auf. Diese sind sehr unregelmäßig in ihrer Form und Größe. Stellenweise treten sie sehr dicht nebeneinander, teilweise aber auch vereinzelt in ansonsten periglaziär unbeeinflussten Till-Bereichen auf.

An der Oberfläche des Aushubbereiches sind bis zu mehr als $10 \mathrm{~m}$ lange, sandgefüllte Spalten zu beobachten, die als schmale Bänder rötlich gefärbter Sande auftreten. Diese sind meist zentral in den Rinnen-Polygonen positioniert (Abb. 4, 6) und häufig in ihrem Verlauf unterbrochen (Abb. 3, 4). Die Breite ist variabel, sie erreicht im Maximum aber ca. 0,15 m. Die Spalten setzen sich keilförmig in die Tiefe fort (Abb. 6). Die Rotfärbung geht auf die Verunreinigungen mit Schadstoffen (PAK), bzw. auf eine Oxidation und chemische Umbildung derselben am Standort zurück. Entsprechende Risse sind auch außerhalb der Rinnen-Polygone im anstehenden Till vorhanden.

An der Unterseite der Rinnen-Polygone setzen teilweise vertikale Risse oder Klüfte an, die bis in mehrere $m$ Tiefe zu verfolgen sind (Abb. 7, 8, 9). Es konnten maximale Teufen der Klüfte von ca. $3 \mathrm{~m}$ unterhalb der Oberkante des Tills nachgewiesen werden. Der weitere Verlauf in die Tiefe konnte unterhalb der Auskofferungssohle naturgemäß nicht geklärt werden. Die Risse zeigen Füllungen aus schluffig-sandigem Material und sind durch Eisenausfällungen braun gefärbt. Die Breite der Rissfüllungen liegt bei maximal wenigen Zentimetern. Die Spalten besitzen eine relativ gleichförmige Breite über die gesamte Tiefe hinweg. Diese Klüfte dürften in einer späten Phase des 


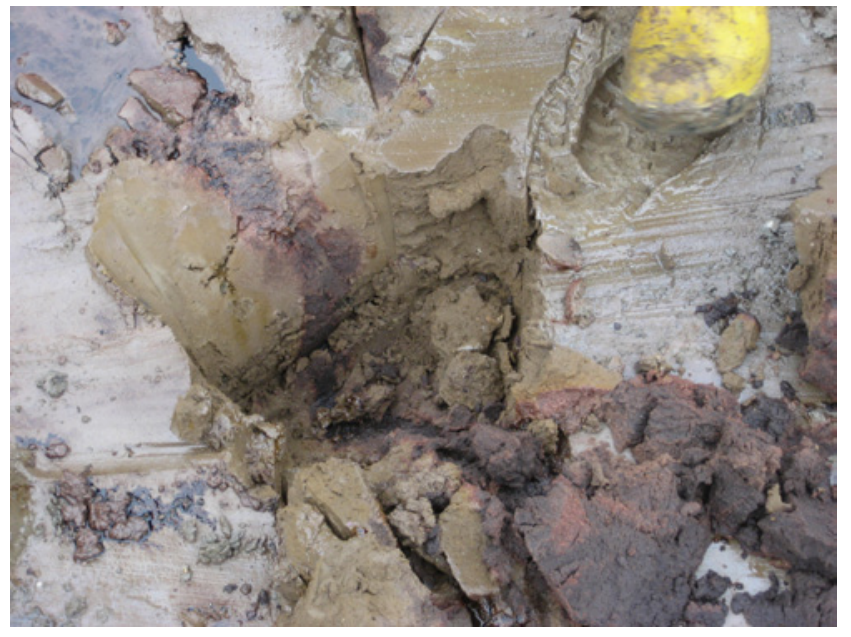

Abb. 6: Fortsetzung der zentral in den Rinnenstrukturen angeordneten rötlichen Sande in die Tiefe (durch Schadstoffe farblich verändert; Stiefelspitze als Maßstab).

Fig. 6: Continuation of the central oriented red sands into depths (coloured by pollutant, boot for scale).

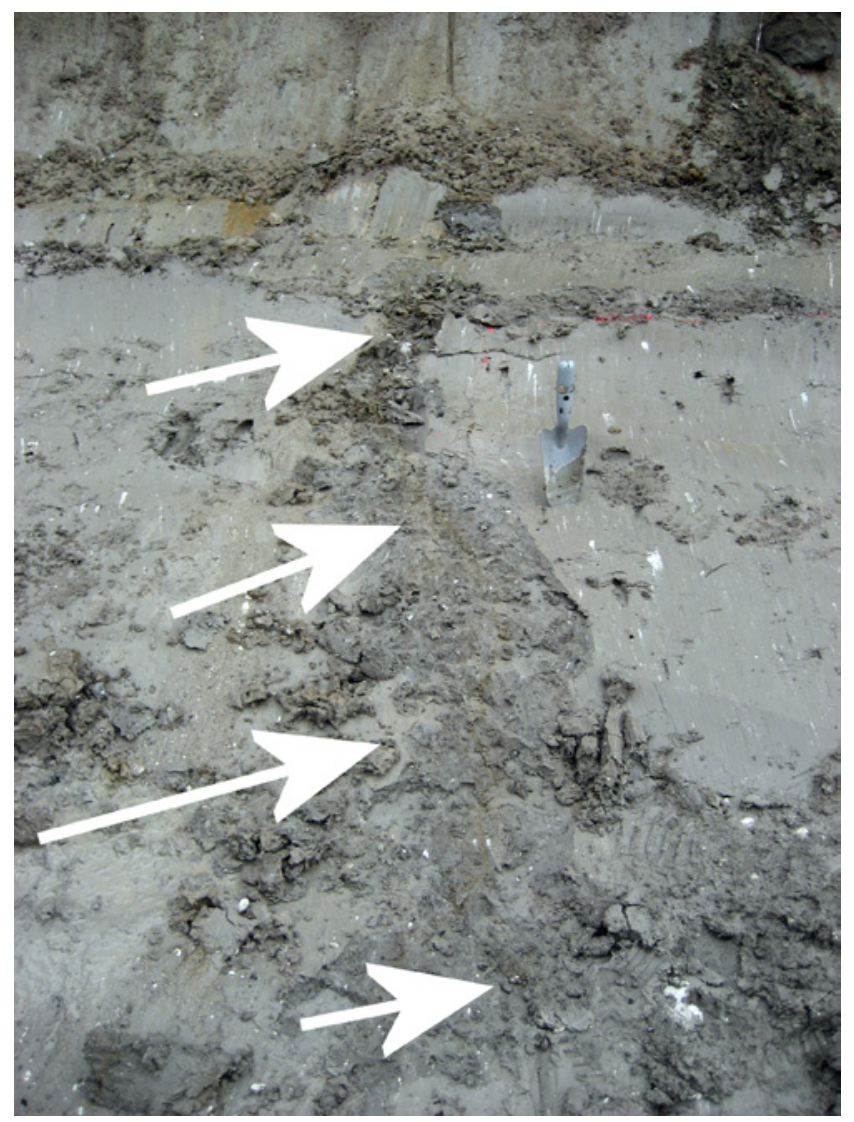

Abb. 7: Wandaufsicht mit Frost-/Eiskeilstruktur, bzw. glazitektonischer

Kluft an der Ostseite des Aufschlusses, maximal wenige cm breit. Farbänderung im umgebenden Till (braune Farbe). Kelle als Maßstab (ca. $25 \mathrm{~cm}$ ) in abgeschrägter Aufschlusswand.

Fig. 7: Frost/ice wedges resp. glacitectonic joints at east site of excavation, max. few cm wide. Colour alteration in surrounding till (brown colour).

Dipper for scale (ca. $25 \mathrm{~cm}$ ) in sloped excavation cut.

Niendorfer Stadiums oder bei der Überfahrung des Bereiches durch einen Gletscher während der jüngeren Saale (Fuhlsbüttler Stadium) glazitektonisch angelegt worden

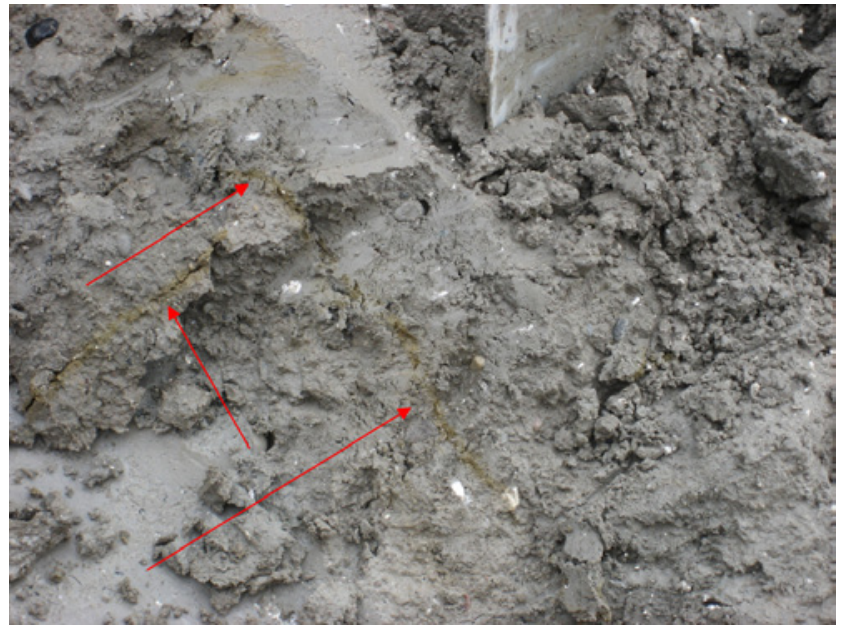

Abb. 8: Frost-/Eiskeilstrukturen, bzw. glazitektonische Klüfte, sich verzweigend, wenige $\mathrm{mm}$ breit, in fast senkrechter Wand (Spaten als Maßstab).

Fig. 8: Frost/ice wedges resp. glacitectonic joints, branched, $\mathrm{mm}$-sized, in nearly vertical wall (spade for scale).

sein. Die Ablagerungen dieses jüngsten Eisvorstoßes sind nur örtlich im Bereich westlich der Blankeneser Berge erhalten geblieben, nicht jedoch im engeren Untersuchungsgebiet.

\section{Hydrogeologie}

Das Grundwasserleitersystem ist gekennzeichnet durch einen hangenden, freien Grundwasserleiter, einen liegenden, in der Matrix geringdurchlässigen Till mit Kluftsystem, einen darunter folgenden, geringmächtigen Grundwasserleiter sowie einen liegenden Till und andere ältere Ablagerungen (Abb. 2). Die Rinnen-Polygone fungieren aufgrund ihrer Lage an der Sohle des oberen Grundwasserleiters, eingeschnitten in den geringer durchlässigen Till, und der im Vergleich zur Umgebung etwas höheren hydraulischen Leitfähigkeit bevorzugt als Sammelbereiche für die Schadstoffe (Abb. 6). Über die zumindest teilweise an der Unterkante der Rinnen-Polygone ansetzenden, bis $\mathrm{zu}$ einer Tiefe von ca. $3 \mathrm{~m}$ unterhalb der Sohle des Aufschlusses nachgewiesenen, sandig-schluffig gefüllten, Klüfte (Abb. 7 und 8), kann sich unter heutigen Bedingungen, trotz geringer Kluftbreite, Grundwasser tief in den Till bzw. Stauerbereich hinein bewegen. Ein Beleg für die Grundwasserbewegung sind die Eisenausfällungen an den Klüften. Wahrscheinlich existieren tiefreichende Strukturen, die den gesamten oberen Till durchschlagen und so zur Verunreinigung des liegenden Grundwasserleiters mit Schadstoffen aus dem oberen Grundwasserleiter geführt haben dürften. Ein analoges Beispiel wurde im Rahmen von Altlastuntersuchungen aus dem Bereich Hamburg-Othmarschen dokumentiert. Dort sind Aromaten aus den oberen Bodenbereichen in den ersten abgedeckten Grundwasserleiter gesickert (freundl. Mitt. Dr. T. Vollmer †, BGW, Hamburg). Dies erfolgte über schmale, glazitektonisch angelegte Klüfte, die im Aufschluss bis zu $7 \mathrm{~m}$ unter die Geländeunterkante in einem $15 \mathrm{~m}$ mächtigen Drenthe-Till nachgewiesen werden konnten. 


\section{Zusammenfassung und Diskussion verschiedener Hypothesen zur Genese der Rinnen-Polygone 5.1 Zusammenfassung der Feldbeobachtungen}

Bei den schmalen, tief in den Till reichenden Störungsstrukturen (Abb. 7, 8) handelt es sich, zumindest im tieferen Teil, um glazitektonisch angelegte Klüfte. Hierfür spricht die durchgehend meist einheitliche, geringe Breite der Klüfte, ihre räumliche Verteilung sowie die rechtwinkligen Verzweigungen (Abb. 7). Über die Verbreitung und Orientierung des glazitektonisch angelegten Kluftnetzes existieren keine Informationen. Glazitektonische Klüfte, d. h. Spalten, die durch den Druck des Inlandeises - vor oder unter dem Gletscher - hervorgerufen werden, können gewöhnlich in allen Lockergesteinsmaterialien des Norddeutschen Flachlandes beobachtet werden, wie bereits erwähnt auch in Wedel. Unterhalb der hangenden Rinnen-Polygone könnte eine Überprägung der Klüfte in Form von Eis-/Frostkeilbildung stattgefunden haben. Sie setzen gewöhnlich an der Oberfläche eines Tills oder einer anderen bindigen Schicht an und nicht wie hier an der Basis der Rinnen-Polygone. Die Spalten besitzen meist eine relativ gleichförmige Breite zur Tiefe hin, was ebenfalls untypisch für eine Eiskeil-/ Frostkeil-Bildung im Permafrost ist. Nach Vandenberghe (1992) sind Polygonnetze von Kryoturbationen normalerweise klein, ihr Durchmesser entspricht etwa der vertikalen Amplitude.

Die in Wedel an der Oberfläche des Tills auftretenden Rinnen-Polygone sind netzartig angeordnet. Es handelt sich nicht um tiefreichende keilförmige Strukturen, sondern um symmetrische, flache, rinnenartige Formen. Bei der Füllung dürfte es sich um aufgearbeitetes Till-Material mit eingearbeitetem, feinkörnigem Sand, teilweise Flugsand, handeln. Der Ursprung der Sandkomponente ist unklar. Am ehesten dürften ehemals geringmächtige, den Till überlagernde Sande eingearbeitet worden sein. Die Füllung der Rinnen-Polygone ist durch einen höheren Feinsandanteil charakterisiert, daher erscheint des Weiteren eine äolische Flugsandkomponente wahrscheinlich. Zudem könnte Material über die Klüfte aus dem Liegenden zugeführt worden sein.

\subsection{Bevorzugte Vorstellung einer polygenetisch bedingten Entstehung der Rinnen-Polygone}

Die Rinnen-Polygone in Wedel dürften unter Frostbodenbedingungen gebildet worden sein. Denkbar erscheint eine Entstehung im Zusammenhang mit den während des Periglazials der Weichsel-Kaltzeit herrschenden artesischen Grundwasserverhältnissen im Randbereich des Elbe-Urstromtales. Die im zweiten (ersten abgedeckten) Grundwasserleiter nachgewiesenen PAK-Kontaminationen weisen auf einen bis in diesen reichenden glazialtektonischen Einfluss, bzw. auf eine Ausdehnung entsprechender Klüfte hin, die hydraulische Wegsamkeiten zwischen dem oberen, freien und dem zweiten Grundwasserleiter bilden. Einen Beleg für die (zeitweise) hydraulische Aktivität der Klüfte stellen die enthaltenen Sandfüllungen und Sandbeläge dar. Eisen-Ausfällungen an den Klüften sind Zeugnisse jüngerer, bzw. aktueller Grundwasserbewegung. Die für eine vertikal aufwärts gerichtete Grundwasserbewegung not- wendigen hydraulischen Potentiale lassen sich durch einen nahegelegenen Gletscher erklären. Nach den vorliegenden Informationen überliefen die Gletscher der jüngeren Saale die Blankeneser Berge, in deren Vorfeld entsprechend teilweise sehr hohe hydraulische Drucke in den Grundwasserleitern geherrscht haben dürften (VAN WEERT et al. 1997). Dabei könnten die besonderen periglaziär-hydraulischen Verhältnisse im Randbereich zum benachbarten Talik des Elbe-Urstromtales eine Rolle gespielt haben. Große Talbereiche bildeten im Permafrost, dessen Mächtigkeit im Hochglazial nach Delisle, CAspers \& Freund (2003) bis zu $140 \mathrm{~m}$ betrug, bekanntlich Taliks (Yershov 1998). Im Randgebiet rezenter Taliks, im Bereich großer Täler, wurden Quellaustritte beschrieben (Kovalenko 1964). Nach Yershov (1998) ist ein Vorkommen von Grundwasser auBerhalb der Talsohle, im Bereich der seitlichen Terrassen, häufig. Cederstrom et al. (1953) erwähnen verschiedene Spaltenartige Wegsamkeiten, wie Klüfte, Spalten, Störungszonen, als mögliche Bewegungsbahnen im aktiven Permafrost. Talik-Verhältnisse können auch für das ElbeUrstromtal während der Periglazial-Zeit angenommen werden. Die randlich hoch gelegenen Nachbarbereiche des Urstromtales waren dagegen durch Permafrostboden charakterisiert. Um die notwendigen Druckverhältnisse für einen Austritt gespannten Grundwassers ca. 8-10 m über der heutigen Oberfläche des Elbtales zu erreichen, muss der Tal-Bereich plombiert sein. Vorstellbar ist eine entsprechende Plombierung während Winterperioden, in denen das Tal zugefroren war.

Der Aufstieg der Grundwässer aus dem Talik-Bereich des Urstromtales erfolgte nach dieser Vorstellung über das vorhandene glazitektonisch gebildete Kluftnetz. Die verhältnismäßig große Mächtigkeit des Tills könnte für die Aufrechterhaltung dieser hydraulischen Bedingungen begünstigend gewirkt haben. Aufwärts gerichtete Gradienten im Grundwasser führten zum Austritt dieses Wassers an der Till-Oberfläche sowie zu einem örtlichen Auftauen des weichselkaltzeitlichen Dauerfrostbodens (Abb. 9). Entlang der linearen Entwässerungsstrukturen, bzw. Austrittsstellen des Grundwassers bildeten sich lokale, im Anschnitt trichterförmig nach oben geöffnete Auftaubereiche im umgebenden Permafrostboden. Hier kam es zur Materialdurchmischung und zur Einmischung von hangenden Sanden sowie von Flugsanden.

Der Prozess der Durchmischung, der eine verhältnismäßig homogene Füllung hervorrief, ist unklar. Denkbar erscheint eine Übersättigung des Porenwasserdruckes (VAndenberghe 1983, 1992). Diese würde durch den aktiven Zustrom von Grundwasser aus dem Liegenden über die Klüfte verstärkt. Bei Vorhandensein einer hangenden Frostfront wären hier besonders günstige Vorrausetzungen für eine kryoturbate Durchmischung gegeben, bei vollständiger Auflösung eines Kontaktes der die Rinnenfüllung bildenden Mineralkörner. Aufgrund der verhältnismäßig homogenen Rinnenfüllung, bzw. der guten Durchmischung dürfte von einer längeren Durchmischungsphase auszugehen sein. Eine laterale Verlagerung, z. B. durch Solifluktion oder Abluation, kann aufgrund der Homogenität des Materials keine große Rolle gespielt haben.

Die Anlage von Rinnen-Polygonen konnte bevorzugt dort erfolgen, wo Schwächezonen in Form von durch 
Tab. 1: Vereinfachte Darstellung der Bildungsphasen von Ablagerungen und Strukturen im Untersuchungsgebiet.

Tab. 1: Simplified representation of the genesis of deposits and structures in the study area.

\begin{tabular}{|c|c|c|c|}
\hline Petrographie & Mächtigkeit & Alterseinstufung & Prozess / Struktur \\
\hline $\begin{array}{l}\text { Rollige und bindige } \\
\text { Ablagerungen, Baustoffe usw. }\end{array}$ & ca. $1-2 \mathrm{~m}$ & Holozän & anthropogene Überprägung und Auftrag \\
\hline Sande & ca. $1-2 \mathrm{~m}$ & Weichsel-Kaltzeit / Frühholozän & Ablagerung von fluviatilen und äolischen Sedimenten \\
\hline Organogene Sedimente & $1-2 \mathrm{~m}$ & Eem-Warmzeit & Sedimentation von Torfen, Mudden, humosen Sanden \\
\hline Sande & ca. $3-4 \mathrm{~m}$ & Jüngere Saale-Kaltzeit & $\begin{array}{l}\text { Ablagerung von glazifluv. / kaltzeitlich-fluviatilen } \\
\text { Sedimenten }\end{array}$ \\
\hline $\begin{array}{l}\text { periglazial überformte } \\
\text { Tilloberfläche }\end{array}$ & ca. $1 \mathrm{~m}$ & $\begin{array}{l}\text { Saale-Kaltzeit; Überformung } \\
\text { während Weichsel-Kaltzeit }\end{array}$ & Bildung der Rinnen-Strukturen \\
\hline nicht erhalten [Till] & n. b. & Jüngere Saale-Vereisung & $\begin{array}{l}\text { glazitektonische Stauchung, bevorzugt des Tills der } \\
\text { Mittl. Saale, Bildung eines Kluftnetzes }\end{array}$ \\
\hline Sande [nicht klar abgrenzbar] & gering & Jüngere Saale-Kaltzeit & ggf. glazifluv. Sedimentation \\
\hline Till [2], ggf. Sande & $6-9 m$ & Mittl. Saale-Vereisung & glazigene Sedimentation, ggf. glazifluv. Sedimentation \\
\hline Sande & $1-2 \mathrm{~m}$ & Ältere / Mittl. Saale & glazifluv. Sedimentation \\
\hline Till [1] & $>5 \mathrm{~m}$ & Ältere Saale-Vereisung & glazigene Sedimentation \\
\hline
\end{tabular}

Frost-/Eiskeilbildung überprägten glazialtektonischen Klüften vorhanden waren. Die Orientierung der RinnenPolygone wurde durch die glazialtektonische Vorzeichnung des Bereiches beeinflusst, worauf das Ansetzen der Strukturen an der Basis der Rinnen-Polygone hindeutet. Das bedeutet, dass die Rinnen-Polygone bevorzugt im Bereich des vorhandenen Kluftnetzes gebildet worden sind. Als Arbeitshypothese wird daher eine „endogene“ Initialisierung der Strukturen favorisiert.

Die zentralen Spaltenfüllungen mit Sand reichen, wie bereits erwähnt, bis an die Sohle der Rinnen-Polygone. Hierbei könnte es sich um die Kontaktbereiche zu den Klüften im Till handeln. Diese kommen naturgemäß nicht in allen Netzteilen vor, da die Klüfte nur Teile des Netzes „abdecken “. Auch dieses spricht für eine hydraulische Aktivität der Klüfte. Der linienhafte Grundwasseraustritt entlang des glazitektonischen Kluftnetzes könnte zur Bildung der sandigen Spaltenfüllungen geführt haben, die im Zentralbereich einiger Rinnenstrukturen verlaufen. Das sandige, heute - anthropogen bedingt - durch Ausfällung von Eisen und organischen Stoffen intensiv rot gefärbte Material, könnte aus dem Bereich des ersten bedeckten Grundwasserleiters ausgetragen worden sein - also eine späte Phase des Grundwasserzutritts über die glazitektonischen Klüfte darstellen oder aus sandigen Ablagerungen oberhalb des Tills stammen. Andererseits ist bei den Spaltenfüllungen eine Bildung als Eiskeil- oder Frostkeil-Pseudomorphose in einer späteren Phase ebenso denkbar.

Eine Initialisierung einer periglaziären Überprägung, bzw. Sub-Permafrost-Entwässerung durch ein existierendes glazialtektonisch angelegtes Kluftnetz als Hypothese der Genese der Rinnen-Polygone würde auch erklären, weshalb entsprechende Strukturen bisher nicht beschrieben worden sind. Diese wären an die unmittelbaren Talrandbereiche oder Tal begleitenden Hochflächen gebunden, die zudem glazialtektonisch gestört sein müssen, um

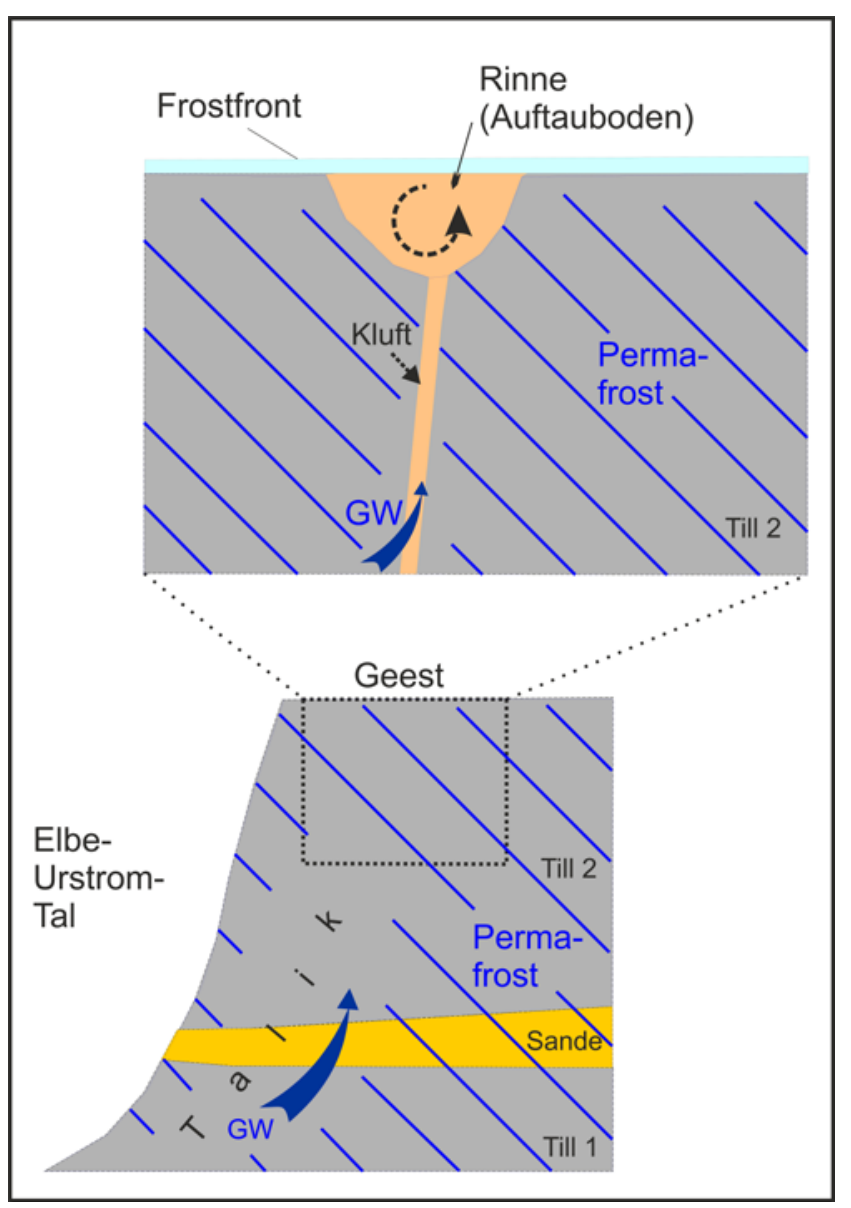

Abb. 9: Schematisierte Zeichnung zu den möglichen Bildungsbedingungen der Rinnen-Bildungen am Rand des Elbe-Urstromtales.

Fig. 9: Scheme showing the possible forming conditions of polygonal channelized structures at the rim of the Elbe spillway. Vgl. Tab. 1. 
entsprechende hydraulische Wegsamkeiten zu schaffen. Einen Einfluss von glazitektonisch beeinflussten Standorten auf geomorphologische Formen beschreibt BER (1986), jedoch in einem erheblich größeren Maßstab. Dieser Autor wies ein polygonartiges Netz von Talformen im Nordosten Polens nach, welches er auf die Erosion von glazitektonisch entstandenen, weniger kompaktierten Zonen zurückführt.

\subsection{Alternative Entstehungsursachen: Periglaziale Frostmusterböden, Involutionen und Keile}

Über Frostmusterböden und Keilstrukturen auf Tilloberflächen und anderen bindigen Sedimenten ist in der Literatur verhältnismäßig wenig berichtet worden. Tatsächlich werden periglaziäre Strukturen bevorzugt in feinkörnigen Ablagerungen, darüber hinaus in schlecht entwässerten Bereichen, gebildet. Nach FrencH (2007) ist die Tatsache, dass in der Literatur häufiger Eiskeil-Pseudomorphosen in sandigen Ablagerungen beschrieben werden, auf die bessere Erhaltungsfähigkeit der Strukturen in diesem Wirtsgestein beim Abtauen des Permafrostes zurückzuführen. Keil-Bildungen sind demnach Bereiche bevorzugter Erosion und Rutschmassenbildung in der Degradationsphase des Permafrostes („Mollisol“, vgl. EISSMANN 1978).

JoHnsson (1959) zeigt taschenförmige, mit sandig-kiesigem Material gefüllte Gebilde, angelegt in sandig-kiesigem Material, die auf Kyroturbation und Solifluktion zurückgehen. Diese sind jedoch deutlich größer und unregelmäßiger ausgebildet als die Strukturen in Wedel. MöRNER (1972) und Humlum (1978) beschreiben „Till-Keile“ (till-wedges) aus Schweden und Dänemark, die subglazial in Reibungsklüften an der Basisfläche eines Tills gebildet werden und meist in sandigem Material angelegt sind. Diese Strukturen weisen jedoch eine deutliche horizontale Komponente auf und sind unregelmäßig geformt.

Teilweise sind Ähnlichkeiten der hier beschriebenen Strukturen mit „Frosttaschen“, bzw. „Lehmsträngen“ (STEEGER 1944, LiEDTKE 1957/58, Golte \& HeINE 1974, EissmanN 1981) vorhanden, die am Niederrhein und in der Leipziger Bucht beobachtet wurden. Bei diesen handelt es sich um „1-3, maximal $5 \mathrm{~m}$ breite und 1-2, maximal $3 \mathrm{~m}$ mächtige, schwere Körper aus kiesigem Lehm und Schluff..." (EIssMANN 1981). Sie können netzartig verbunden sein. Diese auch als „Riesen-Eiskeilnetze“ bezeichneten Strukturen werden jedoch eher taschen-, als rinnenartig beschrieben. Zudem weicht ihre Füllung von den hier beschriebenen Rinnen-Polygonen ab, da diese heterogener aufgebaut ist. Insgesamt weist ihre Form und Füllung in Richtung Kryoturbation, die unregelmäßige Form deutet auf eine Bildung während des Zerfalls des Permafrostbodens hin. Ähnlichkeiten mit den Strukturen aus Wedel sind gegeben in Bezug auf die Größe der Formen und das Vorkommen in bindigem Substrat, wenngleich sie nicht in Till eingeschnitten sind.

DEwers (1934) dokumentierte taschenförmige Strukturen mit feinsandiger Füllung in tonigem Schluffsand (Osterholz-Scharnbek) sowie Taschenböden mit feinsandiger Füllung in Till (Haselünne), die allerdings im Vergleich zu den hier beschriebenen Strukturen deutlich unregelmäßiger geformt sind und vom Autor als Stauchungsstrukturen interpretiert werden. LAskowskA (1960) beschreibt unregelmäßige, topfförmige Taschenbildungen, die mit gut bis schlecht sortierten Sanden gefüllt sind. Sie sind demnach als Kontraktionsstrukturen mit Überformung durch Kryoturbation zu interpretieren. Diese sind jedoch sehr unregelmäßig geformt und bevorzugt punktuell ausgebildet, im Gegensatz zu den hier beschriebenen Formen, die linienhaft und regelmäßig auftreten. JoHnsson (1963) zeigt rundlich-konzentrische, „muschelförmige“ Strukturen (Sandlagen in Ton), die als Frosttaschen interpretiert werden. Eine erosiv gekappte derartige Struktur hätte Ähnlichkeit mit den Rinnen-Polygonen in Wedel. Diese „Sandbälle“ sind jedoch stark intern gegliedert und treten unregelmäßiger verbreitet auf als die Strukturen in Wedel. SoloviEw (1973) bearbeitete 2-5 m große Depressionen, die auf Kryokarst zurückgehen, AKERMAN (1981) Thermokarsthohlformen auf Spitzbergen. Auch diese Strukturen haben nur oberflächliche Ähnlichkeit mit den hier diskutierten RinnenPolygonen.

Bei den hier beschriebenen Formen handelt es sich offenbar nicht um Keilstrukturen. Dennoch ist teilweise eine gewisse Ähnlichkeit mit den in der Literatur beschriebenen, in Teilen basal rundlich geformten Eis-/Frostkeilstrukturen vorhanden. Zudem sind Keilstrukturen oft an Polygonnetze gebunden, die auch in Wedel beobachtet werden können. Daher werden bei der vorliegenden Diskussion Keilstrukturen beim Vergleich mit berücksichtigt. LEFFINGWELL (1915) beschreibt breite Eiskeil-/Frostspaltenformen in Alaska. Romanovskij (1973) bearbeitet verschiedene Eiskeil-Typen, die eine rundliche Ausformung am unteren Ende aufweisen. Diese sind teilweise in Löss-artigen Sedimenten angelegt und mit Schluff gefüllt. GHysels \& HEysE (2006) beschreiben kompliziert zusammengesetzte Keilstrukturen in Flandern / Belgien. Hier wurden auch lang gestreckte, topfförmige Strukturen gefunden. Die dort beschriebenen Formen werden als Frostrisse interpretiert. Es ist eine generelle Ähnlichkeit mit den in Wedel gefundenen Strukturen erkennbar, jedoch weisen sie im Vergleich $\mathrm{zu}$ den hier beschriebenen Formen eine unregelmäßigere Form sowie eine heterogenere und laminierte Füllung auf. Die Anlage der belgischen Formen erfolgte in bindigen Ablagerungen, die allerdings in Bezug auf die Konsistenz deutlich von dem Till in Wedel abweicht.

VANDENBERGHE (1992) bearbeitete Eiskeilnetze auf Tonsedimenten in den südlichen Niederlanden. VANDENBERGHE \& KRоOK (1981) zeigen kyoturbat überformte obere Teile von Eiskeilpseudomorphosen, die eine rundliche Gestalt aufweisen. Auch die hangenden Dezimeter der Eiskeile von Lieth (GRUBE 2007) weisen eine deutliche Verbreiterung und kryoturbate Überformung auf. LAKowsKA (1960) beschreibt ein polygonales Netz auf Till in einem Talbereich bei Gabin (Polen). Die zu dem länglichen, pentagonal geformten Netz gehörenden Spalten sind je nach Position netzförmig ausgebildet. Sie weisen ebenfalls rundliche Formen auf. Einfache Strukturen, d. h. periglaziäre Eiskeile/ Frostrisse sowie Trockenrisse, wurden meist als fossile Formen von BALLANTYNE \& MATTHEws (1983) auf Till in Norwegen, von LagerLund (1980) und Svensson (1987) auf Till in Südschweden, von DzIEREK \& StaÑczUK (2006) auf geringmächtigem, sandbedecktem Till in Ost-Polen sowie von Kabala \& ZAPART (2009) auf marinen Feinsanden (raised shore) in Spitzbergen, von BLACK (1973) auf Till in der Antarktis (Victoria Land) sowie von KATAsONOV 
(1973a, b) auf lössartigen Sedimenten an der Mittleren Lena beschrieben. WALTERS (1994) untersuchte Spaltenbildungen in Iowa (USA), die partiell in Till-Material angelegt waren. Diese sind teils aus einer sehr heterogenen Füllung aus meist sandigem sowie bindigem Material, teilweise mit horizontal gelagerten Sanden gefüllt. Die äußere Form der Spaltenbildungen ist sehr unregelmäßig und die Unterkante liegt teilweise gerundet vor. Sie weisen eine durchschnittliche Breite von ca. $100 \mathrm{~cm}$ sowie eine Tiefe von ca. $185 \mathrm{~cm}$ auf. VANDENBERGHE (1983) beschreibt rundliche Involutionen, die unabhängig von Eiskeilpseudomorphosen gebildet wurden. All diese Strukturen weisen deutliche Unterschiede zu den hier vorgestellten Formen auf.

Auch in Deutschland wurden entsprechende Arbeiten durchgeführt. WORTMANN (1956) dokumentiert Eiskeilnetze in Geschiebelehm im Münsterland, SoERgEL (1936) in bindigem Material (Löss, Lehme) in Mitteldeutschland. BACKHAus (1970) belegt ein mit Löß gefülltes, teilweise rechtwinkliges Eiskeilnetz in Tonschiefern des Lias im Bereich Göttingen-Kassel. In Norddeutschland bearbeiteten u. a. Dücker (1954), Arnold (1955), PiCARd (1956) und Blume, Hoffmann \& Pachur (1979) Eiskeile sowie zugehörige Strukturen. LiEDTKE (1957/58) beschreibt Eiskeile in Geschiebedecksand und Till im südöstlichen Brandenburg (Bornow), in Tonsedimenten bei Plau (Mecklenburg-Vorpommern) sowie an weiteren Standorten. Die oberen Bereiche des miozänen Glimmertons von Groß Pampau zeigen bis zu mehr als $15 \mathrm{~m}$ tief reichende Eiskeile (vgl. HöPFNER 2014). Keilförmige Strukturen konnten vom Autor im Rahmen der Landesaufnahme auch auf den freigelegten Till-Flächen des Gewerbegebietes Süd in Elmshorn/Grauer Esel (Neubau Möbelhaus Kibek 2005) und Bönningstedt (Neubau Fa. Krümet 2005) dokumentiert werden. Diese waren dort jedoch nicht regelmäßig polygonartig vergesellschaftet. Regelmäßige Polygonnetze in Till der mittleren Saale wurden in Itzehoe-Süd (Bau der Autobahnüberführung BAB23 - Abfahrt Itzehoe-Süd 2012) dokumentiert. Bei all diesen Formen handelt es sich jedoch um tiefreichende, keilförmige Strukturen, die meist mit sandigem bis bindigem Material verfüllt sind. Frostkeile, bzw. Eiskeilnetze auf mächtigen Geschiebedeck- und Flugsanden sowie Solifluktionsmaterial werden weiterhin von Hassenpflug (1988, 2013) aus Tinningstedt-Neulandshof und weiteren Standorten beschrieben. Zusammenfassend kann gesagt werden, dass einige der o. g. in der Literatur beschriebenen Strukturen rundliche Ausformungen aufweisen können. All diese Formen sind jedoch i. d. R. eindeutiger vertikallanggestreckt ausgebildet. Ihre Füllung ist zudem überwiegend heterogen und unterscheidet sich deutlich von ihrer Umgebung. Sie sind somit nicht mit den in Wedel dokumentierten Strukturen identisch.

Denkbar ist auch eine Teilerhaltung von Kryoturbationsformen im Till. Die oberen Teile einer entsprechenden Bildung (vgl. Abb. in VANDENBERGHE 1992) wären dann erodiert worden. Diese wäre allerdings auch dann noch rundlicher und nicht so deutlich rinnenförmig ausgebildet, wie die Formen in Wedel. Zudem müssten mächtigere Teile des Tills abgetragen worden sein. Hiergegen spricht jedoch die polygonale Verbreitung der Rinnen gegenüber einer i. d. Regel flächenhaften Ausbildung von Kryoturbationen. Außerdem wäre eine Erosionsfläche ggf. weniger flach ausgebildet.

\subsection{Alternative Entstehungsursache: Bildung als subaerische Struktur}

Generell kann es zu einer Rinnenbildung in Polygonmustern kommen (u. a. EISSMANN 1981, French 2007), wenn beim Austauen von Eiskeilen ein Volumensverlust eintritt. Wenngleich damit gerechnet werden muss, dass die heute weitgehend geschlossenen und nur $\mathrm{mm}$ bis wenige $\mathrm{cm}$ breiten Klüfte im Geschiebemergel im Sinne eines Eiskeils ehemals weiter geöffnet gewesen sein könnten, kann das hierdurch gebildete Volumen jedoch nicht annähernd die Ausmaße der Rinnen-Polygone in Wedel erhalten haben.

Eine Anlage der Rinnen-Polygone als periglaziär-subaerische Bildung, bei der eine Hohlform gebildet wird, die anschließend wieder vollständig verfüllt wurde, ist schwer vorstellbar, vorwiegend wegen der polygonal-strangförmigen Form der Rinnen-Polygone. GRUBE (2011) beschreibt periglaziäre Rinnenstrukturen, die deutlich größer sowie sehr viel heterogener im Aufbau sind und die durch erosivgravitative Abtragungs- bzw. Massenverlagerungs-Prozesse entstanden sein dürften. Die Strukturen in Wedel sind im Vergleich dazu ausgesprochen symmetrisch geformt und mit verhältnismäßig homogenem Material (aufgearbeiteter Till) verfüllt. Eine subaerische Bildung hätte eine Ausräumung und eine anschließende Verfüllung bewirkt, die in jedem Fall deutlich heterogener aufgebaut wäre. Prinzipiell kommt eine Bildung der Strukturen auch als Ergebnis von Hangrutschungen, im Zusammenhang mit Toteisbildungen (kettle cracks, vgl. BLACK 1976) oder als Trockenrisse in Frage. Dieses erscheint jedoch aufgrund des Verlaufes der verzweigten Rinnen-Polygone, der rundlichen Basisfläche und der homogenen Füllung der Strukturen als unplausibel.

\section{Ausblick}

Es ist davon auszugehen, dass die schnelle Abdeckung der Rinnen-Polygone in Wedel durch fluviatile und äolische, saalezeitliche Sande, ggf. auch der Einfluss von Permafrost, die Erhaltung der Formen ermöglicht hat. Weitere Einzelheiten zur Genese, z. B. eine Orientierung des glazitektonischen Kluftnetzes und die räumliche Verbreitung der Strukturen sollten bei zukünftigen Aufschlüssen am Standort Wedel auch durch geophysikalische Methoden näher untersucht werden. OSL-Datierungen wären zur Klärung der stratigraphischen Einordnung der hangenden Sande im Untersuchungsgebiet wünschenswert.

\section{Danksagung}

Der ExxonMobil sei für die Genehmigung zur Publikation der Untersuchung gedankt. Danken möchte ich zudem Frau Dr. C. Kabel-Windloff (LLUR), Frau S. Weik (Fachdienst Umwelt des Kreises Pinneberg), Herr G. Seggelke (Stadt Wedel/Holst.), Herrn S. Seifert (Fa. ARCADIS), Herrn J. Gawenda (Ingenieurbüro ETB Nord) und Herrn A. Klotz (ExxonMobil-Einheit Global remediation) für die Unterstützung bei den Feld-Aufnahmen und Daten-Auswertungen. Herrn Dr. T. Vollmer † (BGW, Hamburg), Herrn K. Schelkes (BGR, Hannover) und Frau C. Siegert (Zittau) danke ich für Hinweise zu vergleichbaren Standorten und Literatur. Herrn W. Lininsch (LLUR) danke ich für die 
Übersetzungshilfe bei der russischen Literatur. Herrn Prof. J. Vandenberghe (VU University Amsterdam) sowie einem weiteren Reviewer danke ich für die Durchsicht des Manuskriptes und kritische Bemerkungen. Die Arbeiten wurden vom Autor im Rahmen seiner Tätigkeit beim Geologischen Dienst Schleswig-Holstein (LLUR) durchgeführt.

\section{Literatur}

Akerman, J. (1981): Studies on Periglacial Geomorphology in West Spitsbergen. - Meddelanden fran Lunds Universitets Geografiska Institution, Avhandlingar, 89: 1-297.

Arnold, H. (1955): Ein aufgedecktes würmzeitliches Frostspalten-Polygonnetz bei Gütersloh. - Zeitschrift deutsche Geologische Gesellschaft, 105: 137.

BAcкhaus, E. (1970): Baugeologie der Lockergesteine (Mittelgebirge, Alpenvorland, Niederrhein). - Grundbautaschenbuch, 1: 55-108.

Ballantyne, C. K. \& Matthews, J. A. (1983): Desiccation Cracking and Sorted Polygon Development, Jotunheimen, Norway. - Arctic and Alpine Research, 15 (3): 339-349.

BER, A. (1987): Glaciotectonic deformation of glacial landforms and de posits in the Suwalki Lakeland (NE Poland). - In: INQUA Symposium on the Genesis and Lithology of Glacial Deposits, Amsterdam, 1986: Tills and Glaciotectonics / ed. by J.J.M. van der Meer. - Rotterdam (Balkema), S. 135-143.

BIG - Prof. BURMEIER Ingenieurgesellschaft mbH (2009): Mineralölwerk Wedel Sanierungsplan. - Bericht i. A. der Stadt Wedel / Holst., 103 S., Gehrden [unveröff.].

BLACK, R. F. (1973): Growth of patterned ground in Victoria Land, Antarctica, in Permafrost. - North American Contribution to the second International Conference, Yakutsk, USSR, National Academy Press, Washington DC, S. 193-203.

BLACK, R. F. (1976). Periglacial features indicative of permafrost: ice and soil wedges. - Quaternary Research, 6: 3-26.

Blume, H.-P., Hoffmann, R. \& Pachur, H.-J. (1979): Periglaziäre Steinring- und Frostkeilbildungen norddeutscher Parabraunerden. - Zeitschrift Geomorphologie, Neue Folge, Supplement-Band, 33: 257-265.

Cenerstrom, D. J., Johnston, P. M. \& Subitzky, S. (1953): Occurrence and development of ground water in permafrost regions. - Geological Survey Circular 275: 130, Washington D.C.

Delisle, G., Caspers, G. \& Freund, H. (2003): Permafrost in north-central Europe during the Weichselian: how deep? - Permafrost: proceedings of the $8^{\text {th }}$ International Conference on Permafrost; S. 187-192, Balkema.

Dewers, F. (1934): Kritische Betrachtungen zur Frage der Beschaffenheit und Entstehung der Taschenböden. - Abhandlungen der Bremer wissenschaftlichen Gesellschaft, 8/9: 306-330.

Dücker, A. (1954): Die Periglazial-Erscheinungen im holsteinischen Pleistozän. - Göttinger Geographische Abhandlungen, 16: 5-54 + Anhang.

DzIEREK, J. \& StAÑCzuK, D. (2006): Record and palaeogeographical implications of Pleistocene periglacial processes in the Drohiczyn Plateau, Podlasie Lowland (Eastern Poland). - Geological Quarterly, 50 (2): 219-228.

EISSMANN, L. (1978): Mollisoldiapirismus. - Zeitschrift angewandte Geologie, 24 (3): 130-138.

Eissmann, L. (1981): Periglaziäre Prozesse und Permafroststrukturen aus sechs Kaltzeiten des Quartärs. - Altenberger Naturwissenschaftliche Forschungen, 1: 1-127, Anhang.

French, H. M. (2007): The Periglacial Environment. - 458 S., 3. Aufl., Chichester (Wiley \& Sons).

Ghysels, G. \& Heyse, I. (2006): Composite-wedge pseudomorphs in Flanders, Belgium. - Permafrost and Periglacial Processes, 17: 145-162.

Golte, W. \& K. Heine (1974): Fossile Riesen-Eiskeilnetze am Niederrhein. - Eiszeitalter und Gegenwart, 25: 132-140.

Grube, A. (2007): Zur Struktur und Entstehung von Eiskeil-Großformen in Lieth / Elmshorn (Schleswig-Holstein). - E\&G Quaternary Science Journal, 56 (4): 283-294.

Grube, A. (2008): Ehemaliges Mineralöl-Werk Wedel der ExxonMobil periglaziäre und glazialtektonische Strukturen. - Interner Bericht, Landesamt für Natur und Umwelt des Landes Schleswig-Holstein; 10 S. + Anhang [unveröff.]

Grube, A. (2011): Zur periglaziären Überformung und der Bildung rinnenartiger Strukturen im Jungmoränen-Gebiet Süd-Holsteins. - E\&G Quaternary Science Journal, 61 (1): 69-83.
GRube, F. (1962): Die Geologie des Schulauer Ufers an der Unterelbe.- In Rust, A. \& Steffens, G. (1962): Die Artefakte der Altonaer Stufe von Wittenbergen: eine mittelpleistozäne Untergruppe der Heidelberger Kulturen. - Neumünster: Wachholtz, Offa-Bücher, 17: 14-24.

Grube, F. (1981): The Subdivision of the Saalian in the Hamburg Region. - Mededelingen - Rijks Geologische Dienst, 34-4: 15-25, Haarlem.

HALliK, R. (1957): Material zur Vegetationsentwicklung während der Eem-Warmzeit in Nordwestdeutschland. - Mitteilungen Geologisches Staatsinstitut Hamburg, 26: 31-38

Hassenpflug, W. (1988): Polygonmuster auf der Schleswiger Geest. Geographische Rundschau, 40 (5): 27-32.

Hassenpflug, W. (2013): Zu Verbreitung und Erscheinungsformen von Polygonmustern in Schleswig-Holstein - eine luftbildgestützte Erfassung. - Natur- und Landeskunde, 120 (4-6): 49-67.

HöPfNER. G. (2014): Aus der Urzeit aufgetaucht. - Berichte des Museums für Natur- und Umwelt und des Naturwissenschaftlichen Vereins zu Lübeck 2a, 182 S, Lübeck.

Humlum, O. (1978): A large till wedge in Denmark: implications for the subglacial regime. - Bulletin Geological Society Denmark, 27: 63-71

Johnsson, G. (1959). True and false ice-wedges in southern Sweden. Geografiska Annaler, 41: 15-33.

Johnsson, G. (1963): Periglacial Phenomena in Southern Sweden. - I. Stratigraphy. - Lund studies in geography, A21: 378-404.

Kabala, C. \& Zapart, J. (2009): Recent, relic and buried soils in the forefield of Werenskiold Glacier, SW Spitsbergen. - Polish Polar Research, 30 (2): 161-178.

Katasonov, E. M. (1973a): Classification of frost-caused phenomena with references to the genesis of the sediments in central Yakutia. - Biuletyn Peryglacjalny, 23: 71-80.

Katasonov, E. M. (1973b): Present-day ground- and ice veins in the region of the Middle Lena. - Biuletyn Peryglacjalny, 23: 81-89.

Kovalenko, V. Ya. (1964): The subpermafrost water of the right bank of the upper basin of the Bol'shaya Anyui. - Material on the geology and minerals of the N.E. USSR, 17: 190-9. Magadan.

LAGERLUND, E. (1980): Litostratigrafisk indelning av Våstskånes Pleistocene och en ny glaciationsmodell for Weichsel. - University of Lund, Department of Quaternary Geology, Report nr. 21: 1-120.

LAsкowskA, W. (1960): Fossil polygonal structures in boulder clay. Biuletyn Peryglacjalny, 7: 177-179 + Abb., Lodz.

Leffingwell, K. ( 1915): Ground Ice-Wedges. The dominant form of ground ice on the north coast of Alaska. - Journal of Geology, 23: 635-654.

LiedTKe, H. (1957/58): Frostbodenstrukturen aus dem norddeutschen Jungmoränengebiet. - Wissenschaftliche Zeitschrift der HumboldtUniversität zu Berlin, Mathematisch-Naturwissenschaftliche Reihe, VII (3): 359-376.

MÖRNER, N.-A. (1972): The first report on till wedges in Europe and Late Weichselian ice flows over southern Sweden. - Geologiska Föreningen Förhandlingar, 94: 581-587, Stockholm.

Nitsche, J. (2012): Geologie und Hydrogeologie des Bereiches östliches Wedel / Hamburg Rissen. - Diplomarbeit (Teile I u. II)., 83 S., Anhang; Dept. Geowiss., Hamburg [unveröff.].

PiCARD, K. (1956): Eiskeile bei Kellinghusen (Mittelholstein). - Neues Jahrbuch Geologie Paläontologie, Monatshefte, 1956: 365-373.

RomanovskiJ, N. N. (1973): Regularities in formation of frost-fissures and development of frost-fissure polygons. - Biuletyn Peryglacjalny, 23: 237-277.

Soergel, W. (1936): Diluviale Eiskeile. - Zeitschrift deutsche Geologische Gesellschaft, 88: 223-247

Soloview, P. A. (1973): Thermokarst phenomena and landforms due to frost heaving in central Yakutia. - Biuletyn Peryglacjalny, 23: 135155.

SteEger, A. (1944): Diluviale Frostbodenerscheinungen am Niederhein. Geologische Rundschau 34: 520-538.

Stephan, H.-J. (1995): Schleswig-Holstein. - In: BendA, L. [Hrsg.]: Das Quartär Deutschlands: S. 1-22, Berlin-Stuttgart (Borntraeger).

SvENSSON, H. (1987): Ice-wedge casts in an early deglaciated area of southern Sweden. - Geografisk Tidsskrift, 87: 12-19.

VANDENBERGHE, J. (1983): Some periglacial phenomena and their stratigraphical position in Weichselian deposits in the Netherlands. - Polarforschung, 53 (2): 97-107.

VANDENBerghe, J. (1992): Cryoturbations: A Sediment Structural Analysis. - Permafrost and Periglacial Processes, 3: 343-352.

VANDENBERGHe, J. ¿ KRooK, L. (1981): Stratigraphy and genesis of Pleistocene deposits at Alphen (southern Netherlands). - Geologie en Mijnbouw, 60: 417-426. 
VAn Weert, F.H.A., VAn Gijssel, K., Leijnse, A. \& Boulton, G.S. (1997) The effects of Pleistocene glaciations on the geohydrological system of Northwest Europe. - Journal of Hydrology 195: 137-159.

Walters, J. C. (1994): Ice-Wedge Casts and Relict Polygonal Patterned Ground in North-East Iowa, USA. - Permafrost and Periglacial Processes, 5: 269-282.

WASHBURN, A. L. (1979): Geocryrology - a survey of periglacial processes and environments. - London (Edward Arnold Press).

Wilke, H. (1984): Zur Geologie des Raumes Altona, Blankenese, Tinsdal im Westen Hamburgs. - Mitteilungen Geologisch-Paläontologisches Institut Universität Hamburg, 57: 91-121.
Wilke, H. \& EhLers, J. (1984): The thrust moraine of Hamburg-Blankenese. - In: Ehlers, J. (Hrsg.): Glacial Deposits in North-West Europe. S. 331-333, Rotterdam (Balkema).

Wolff, W. (1913): Erläuterungen zur Geologischen Karte von Preußen und benachbarten Bundesstaaten, Blatt Wedel. - Berarb. von Schröder, H., Stoller J. \& Wolff, W.; Königl. Preuss. Geol. LandesanSTALt [Hrsg.], Berlin, 37 S. + Anhang.

WortmanN, H. (1956): Ein erstes sicheres Vorkommen von periglazialem Steinnetzboden im Norddeutschen Flachland. - Eiszeitalter \& Gegenwart, 7 (1): 119-126.

Yershov, E. D. (1998): General Geocryology. - 580 S., Cambridge (Cambridge University Press). 